JOURNAL OF THE

CHUNGCHEONG MATHEMATICAL SOCIETY

Volume 26, No. 4, November 2013

http://dx.doi.org/10.14403/jcms.2013.26.4.907

\title{
ON THE STABILITY OF A GENERAL ADDITIVE FUNCTIONAL INEQUALITY IN BANACH SPACES
}

\author{
SANG-Cho ChUnG*
}

\begin{abstract}
In this paper, we prove the generalized Hyers-Ulam stability of the additive functional inequality$$
\left\|f\left(2 x_{1}\right)+f\left(2 x_{2}\right)+\cdots+f\left(2 x_{n}\right)\right\| \leq\left\|t f\left(x_{1}+x_{2}+\cdots+x_{n}\right)\right\|
$$

in Banach spaces where a positive integer $n \geq 3$ and a real number $t$ such that $2 \leq t<n$.
\end{abstract}

\section{Introduction}

In 1940, S. M. Ulam [4] suggested the stability problem of functional equations concerning the stability of group homomorphisms.

In the next year, D. H. Hyers [1] gave a first (partial) affirmative answer to the question of Ulam for Banach spaces as follows: If $\delta>0$ and if $f: \mathcal{X} \rightarrow \mathcal{Y}$ is a mapping between Banach spaces $\mathcal{X}$ and $\mathcal{Y}$ satisfying

$$
\|f(x+y)-f(x)-f(y)\| \leq \delta
$$

for all $x, y \in \mathcal{X}$, then there is a unique additive mapping $A: \mathcal{X} \rightarrow \mathcal{Y}$ such that $\|f(x)-A(x)\| \leq \delta$ for all $x, y \in \mathcal{X}$.

This type is called the Hyers-Ulam stability.

Throughout this paper, let $\mathcal{X}$ be a normed linear space and $\mathcal{Y}$ a Banach space. Let $f: \mathcal{X} \rightarrow \mathcal{Y}$ be a mapping. In 2007, C. Park, Y. S. Cho and M. H. Han [3] proved the generalized Hyers-Ulam stability of the additive functional inequality

$$
\|f(x)+f(y)+f(z)\| \leq\|f(x+y+z)\|
$$

in Banach spaces. In 2011, J. R. Lee, C. Park and D. Y. Shin [2] studied the generalized Hyers-Ulam stability of the additive functional inequality

$$
\|f(2 x)+f(2 y)+2 f(z)\| \leq\|2 f(x+y+z)\|
$$

Received September 31, 2013; Accepted October 30, 2013.

2010 Mathematics Subject Classification: Primary 39B82; Secondary 46B06.

Key words and phrases: additive functional inequality, Hyers-Ulam stability, Banach space. 
in Banach spaces.

In this paper, we give some generalized Hyers-Ulam stability of the additive functional inequality

$$
\left\|f\left(2 x_{1}\right)+f\left(2 x_{2}\right)+\cdots+f\left(2 x_{n}\right)\right\| \leq\left\|t f\left(x_{1}+x_{2}+\cdots+x_{n}\right)\right\|
$$

in Banach spaces where $3 \leq n$ and $2 \leq t<n(n \in \mathbb{Z}$ and $t \in \mathbb{R})$.

\section{Hyers-Ulam stability in Banach spaces}

To obtain our main result, we need the following lemma.

Lemma 2.1. Let $f: \mathcal{X} \rightarrow \mathcal{Y}$ be a mapping and let $3 \leq n$ and $2 \leq t<n$ where $n$ is an integer and $t$ is a real number. Then $f$ is additive if and only if it satisfies

$$
\left\|f\left(2 x_{1}\right)+f\left(2 x_{2}\right)+\cdots+f\left(2 x_{n}\right)\right\| \leq\left\|t f\left(x_{1}+x_{2}+\cdots+x_{n}\right)\right\|
$$

for all $x_{1}, x_{2}, \cdots, x_{n} \in \mathcal{X}$.

Proof. If $f$ is additive, then clearly

$$
\left\|f\left(2 x_{1}\right)+\cdots+f\left(2 x_{n}\right)\right\|=\left\|2 f\left(x_{1}+\cdots+x_{n}\right)\right\| \leq\left\|t f\left(x_{1}+\cdots+x_{n}\right)\right\|
$$

for all $x_{i} \in \mathcal{X}$.

Conversely, assume that $f$ satisfies (2.1). Letting $x_{i}=0$ in (2.1), we gain $\|n f(0)\| \leq\|t f(0)\|$ and so $f(0)=0$ by the assumpution. Putting $x_{i}=0$ for all $i=3, \cdots, n$, and replacing $x_{1}, x_{2}$ by $x,-x$ in (2.1), we get $\|f(-x)+f(x)\| \leq\|t f(0)\|=0$ and so $f(-x)=-f(x)$ for all $x \in \mathcal{X}$.

Setting $x_{1}=\frac{x+y}{2}, x_{2}=\frac{-x}{2}, x_{3}=\frac{-y}{2}, x_{i}=0(4 \leq i \leq n)$ in (2.1), we have

$$
\|f(x+y)+f(-x)+f(-y)\| \leq\|t f(0)\|=0
$$

for all $x, y \in \mathcal{X}$. Thus we obtain $f(x+y)=f(x)+f(y)$ for all $x, y \in$ $\mathcal{X}$.

Theorem 2.2. Let $f: \mathcal{X} \rightarrow \mathcal{Y}$ be a mapping with $f(0)=0$ and let $3 \leq n$ and $2 \leq t<n$. If there is a function $\varphi: \mathcal{X}^{n} \rightarrow[0, \infty)$ satisfying

$$
\left\|f\left(2 x_{1}\right)+\cdots+f\left(2 x_{n}\right)\right\| \leq\left\|t f\left(x_{1}+\cdots+x_{n}\right)\right\|+\varphi\left(x_{1}, \cdots, x_{n}\right)
$$

$$
\widetilde{\varphi}\left(x_{1}, \cdots, x_{n}\right):=\sum_{j=0}^{\infty} \frac{1}{2^{j}} \varphi\left((-2)^{j} x_{1},(-2)^{j} x_{2},(-2)^{j} x_{3}, x_{4}, \cdots, x_{n}\right)<\infty
$$


for all $x_{1}, \cdots, x_{n} \in \mathcal{X}$, then there exists a unique additive mapping $A: \mathcal{X} \rightarrow \mathcal{Y}$ such that

$$
\|f(x)-A(x)\| \leq \frac{1}{2} \widetilde{\varphi}\left(x,-\frac{x}{2},-\frac{x}{2}, 0, \cdots, 0\right)
$$

for all $x \in \mathcal{X}$.

Proof. Replacing $x_{1}, x_{2}, x_{3}, x_{i}(4 \leq i)$ by $(-2)^{n+1} \frac{x}{2},(-2)^{n} \frac{x}{2},(-2)^{n} \frac{x}{2}, 0$, respectively, and dividing by $2^{n+1}$ in $(2.2)$, since $f(0)=0$, we get

$$
\begin{aligned}
& \left\|\frac{f\left((-2)^{n+1} x\right)}{(-2)^{n+1}}-\frac{f\left((-2)^{n} x\right)}{(-2)^{n}}\right\| \\
& \leq \frac{1}{2^{n+1}} \varphi\left((-2)^{n+1} \frac{x}{2},(-2)^{n} \frac{x}{2},(-2)^{n} \frac{x}{2}, 0, \cdots, 0\right)
\end{aligned}
$$

for all $x \in \mathcal{X}$ and all nonnegative integers $n$. From the above inequality, we have

$$
\begin{aligned}
& \left\|\frac{f\left((-2)^{n} x\right)}{(-2)^{n}}-\frac{f\left((-2)^{m} x\right)}{(-2)^{m}}\right\| \\
& \leq \sum_{j=m}^{n-1}\left\|\frac{f\left((-2)^{j+1} x\right)}{(-2)^{j+1}}-\frac{f\left((-2)^{j} x\right)}{(-2)^{j}}\right\| \\
& \leq \sum_{j=m}^{n-1} \frac{1}{2^{j+1}} \varphi\left((-2)^{j} x,(-2)^{j-1} x,(-2)^{j-1} x, 0, \cdots, 0\right)
\end{aligned}
$$

for all $x \in \mathcal{X}$ and all nonnegative integers $m, n$ with $m<n$. By the condition (2.3), the sequence $\left\{\frac{f\left((-2)^{n} x\right)}{(-2)^{n}}\right\}$ is a Cauchy sequence for all $x \in \mathcal{X}$. Since $\mathcal{Y}$ is complete, the sequence $\left\{\frac{f\left((-2)^{n} x\right)}{(-2)^{n}}\right\}$ converges for all $x \in \mathcal{X}$. So we can define a mapping $A: \mathcal{X} \rightarrow \mathcal{Y}$ by

$$
A(x):=\lim _{n \rightarrow \infty} \frac{f\left((-2)^{n} x\right)}{(-2)^{n}}
$$

for all $x \in \mathcal{X}$.

In order to prove that $A$ satisfies (2.4), taking $m=0$ and letting $n$ tend to $\infty$ in (2.5), then we have the following inequality (2.4).

$$
\begin{aligned}
\|A(x)-f(x)\| & \leq \sum_{j=0}^{\infty} \frac{1}{2^{j+1}} \varphi\left((-2)^{j} x,(-2)^{j-1} x,(-2)^{j-1} x, 0, \cdots, 0\right) \\
& =\frac{1}{2} \widetilde{\varphi}\left(x,-\frac{x}{2},-\frac{x}{2}, 0, \cdots, 0\right) .
\end{aligned}
$$


Next we show that $A$ is additive. Replacing $x_{i}$ by $(-2)^{n} x_{i}$ for all $i=1,2, \cdots, n$, and dividing by $2^{n}$ in $(2.2)$, we obtain

$$
\begin{aligned}
& \left\|\frac{f\left((-2)^{n} 2 x_{1}\right)}{(-2)^{n}}+\frac{f\left((-2)^{n} 2 x_{2}\right)}{(-2)^{n}}+\cdots+\frac{f\left((-2)^{n} 2 x_{n}\right)}{(-2)^{n}}\right\| \\
& \leq\left\|t \frac{f\left((-2)^{n}\left(x_{1}+x_{2}+\cdots+x_{n}\right)\right)}{(-2)^{n}}\right\| \\
& \quad+\frac{1}{2^{n}} \varphi\left((-2)^{n} x_{1},(-2)^{n} x_{2}, \cdots,(-2)^{n} x_{n}\right)
\end{aligned}
$$

for all $x_{1}, x_{2}, \cdots, x_{n} \in \mathcal{X}$ and all nonnegative integers $n$. Since (2.3) gives that

$$
\lim _{n \rightarrow \infty} \frac{1}{2^{n}} \varphi\left((-2)^{n} x_{1},(-2)^{n} x_{2}, \cdots,(-2)^{n} x_{n}\right)=0
$$

for all $x_{1}, x_{2}, \cdots, x_{n} \in \mathcal{X}$, letting $n$ tend to $\infty$ in the above inequality, we have

$$
\left\|A\left(2 x_{1}\right)+A\left(2 x_{2}\right)+\cdots+A\left(2 x_{n}\right)\right\| \leq\left\|t A\left(x_{1}+x_{2}+\cdots+x_{n}\right)\right\|
$$

so $A$ is additive by Lemma 2.1 .

Let $A^{\prime}: \mathcal{X} \rightarrow \mathcal{Y}$ be another additive mapping satisfying (2.4). Since both $A$ and $A^{\prime}$ are additive, we have, for all positive integer $n$

$$
\begin{aligned}
& \left\|A(x)-A^{\prime}(x)\right\| \\
& =\frac{1}{2^{n}}\left\|A\left((-2)^{n} x\right)-A^{\prime}\left((-2)^{n} x\right)\right\| \\
& \leq \frac{1}{2^{n}}\left(\left\|A\left((-2)^{n} x\right)-f\left((-2)^{n} x\right)\right\|+\left\|f\left((-2)^{n} x\right)-A^{\prime}\left((-2)^{n} x\right)\right\|\right) \\
& \leq \frac{1}{2^{n}} \widetilde{\varphi}\left((-2)^{n} x,(-2)^{n-1} x,(-2)^{n-1} x, 0, \cdots, 0\right) \\
& =\sum_{j=n}^{\infty} \frac{1}{2^{j}} \varphi\left((-2)^{j-n} x,(-2)^{j-1-n} x,(-2)^{j-1-n} x, 0, \cdots, 0\right)
\end{aligned}
$$

which goes to zero as $n \rightarrow \infty$ for all $x \in \mathcal{X}$ by (2.3). Therefore, $A$ is a unique additive mapping satisfying (2.4), as desired.

Theorem 2.3. Let $f: \mathcal{X} \rightarrow \mathcal{Y}$ be a mapping and let $3 \leq n$ and $2 \leq t<n$. If there is a function $\varphi: \mathcal{X}^{n} \rightarrow[0, \infty)$ satisfying

$$
\left\|f\left(2 x_{1}\right)+\cdots+f\left(2 x_{n}\right)\right\| \leq\left\|t f\left(x_{1}+\cdots+x_{n}\right)\right\|+\varphi\left(x_{1}, \cdots, x_{n}\right) .
$$


where

$$
\begin{aligned}
& \widetilde{\varphi}\left(x_{1}, x_{2}, \cdots, x_{n}\right) \\
& :=\sum_{j=1}^{\infty} 2^{j} \varphi\left(\frac{x_{1}}{(-2)^{j}}, \frac{x_{2}}{(-2)^{j}}, \frac{x_{3}}{(-2)^{j}}, x_{4}, \cdots, x_{n}\right)<\infty
\end{aligned}
$$

for all $x_{1}, x_{2}, \cdots, x_{n} \in \mathcal{X}$, then there exists a unique additive mapping $A: \mathcal{X} \rightarrow \mathcal{Y}$ such that

$$
\|f(x)-A(x)\| \leq \frac{1}{2} \widetilde{\varphi}\left(x,-\frac{x}{2},-\frac{x}{2}, 0, \cdots, 0\right)
$$

for all $x \in \mathcal{X}$.

Proof. We have $\varphi(0, \cdots, 0)=0$ by $(2.7)$, and so $f(0)=0$ by (2.6). Replacing $x_{1}, x_{2}, x_{3}, x_{i}(4 \leq i)$ by $\frac{x}{(-2)^{n}}, \frac{x}{(-2)^{n+1}}, \frac{x}{(-2)^{n+1}}, 0$, respectively, and multiplying by $2^{n-1}$ in $(2.6)$, since $f(0)=0$, we get

$$
\begin{aligned}
& \left\|(-2)^{n-1} f\left(\frac{x}{(-2)^{n-1}}\right)-(-2)^{n} f\left(\frac{x}{(-2)^{n}}\right)\right\| \\
& \leq 2^{n-1} \varphi\left(\frac{x}{(-2)^{n}}, \frac{x}{(-2)^{n+1}}, \frac{x}{(-2)^{n+1}}, 0, \cdots, 0\right)
\end{aligned}
$$

for all $x \in \mathcal{X}$ and all nonnegative integers $n$. From the above inequality, we have

$$
\begin{aligned}
& \left\|(-2)^{n} f\left(\frac{x}{(-2)^{n}}\right)-(-2)^{m} f\left(\frac{x}{(-2)^{m}}\right)\right\| \\
& \leq \sum_{j=m+1}^{n}\left\|(-2)^{j} f\left(\frac{x}{(-2)^{j}}\right)-(-2)^{j-1} f\left(\frac{x}{(-2)^{j-1}}\right)\right\| \\
& \leq \sum_{j=m+1}^{n} 2^{j-1} \varphi\left(\frac{x}{(-2)^{j}}, \frac{x}{(-2)^{j+1}}, \frac{x}{(-2)^{j+1}}, 0, \cdots, 0\right)
\end{aligned}
$$

for all $x \in \mathcal{X}$ and all nonnegative integers $m, n$ with $m<n$. By the condition (2.7), the sequence $\left\{(-2)^{n} f\left(\frac{x}{(-2)^{n}}\right)\right\}$ is a Cauchy sequence for all $x \in \mathcal{X}$. Since $\mathcal{Y}$ is complete, the sequence $\left\{(-2)^{n} f\left(\frac{x}{(-2)^{n}}\right)\right\}$ converges for all $x \in \mathcal{X}$. So we can define a mapping $A: \mathcal{X} \rightarrow \mathcal{Y}$ by

$$
A(x):=\lim _{n \rightarrow \infty}\left\{(-2)^{n} f\left(\frac{x}{(-2)^{n}}\right)\right\}
$$

for all $x \in \mathcal{X}$. 
In order to prove that $A$ satisfies (2.8), taking $m=0$ and letting $n$ tend to $\infty$ in (2.9), then we have the following inequality (2.8).

$$
\begin{aligned}
\|A(x)-f(x)\| & \leq \sum_{j=0}^{\infty} 2^{j-1} \varphi\left(\frac{x}{(-2)^{j}}, \frac{x}{(-2)^{j+1}}, \frac{x}{(-2)^{j+1}}, 0, \cdots, 0\right) \\
& =\frac{1}{2} \widetilde{\varphi}\left(x,-\frac{x}{2},-\frac{x}{2}, 0, \cdots, 0\right) .
\end{aligned}
$$

Next we show that $A$ is additive. Replacing $x_{i}$ by $\frac{x_{i}}{(-2)^{n}}$ for all $i=1,2, \cdots, n$, and multiplying by $2^{n}$ in (2.6), we obtain

$$
\begin{aligned}
& \left\|(-2)^{n} f\left(\frac{2 x_{1}}{(-2)^{n}}\right)+(-2)^{n} f\left(\frac{2 x_{2}}{(-2)^{n}}\right)+\cdots+(-2)^{n} f\left(\frac{2 x_{n}}{(-2)^{n}}\right)\right\| \\
& \leq\left\|t(-2)^{n} f\left(\frac{\left(x_{1}+x_{2}+\cdots+x_{n}\right)}{(-2)^{n}}\right)\right\| \\
& \quad+2^{n} \varphi\left(\frac{x_{1}}{(-2)^{n}}, \frac{x_{2}}{(-2)^{n}}, \cdots, \frac{x_{n}}{(-2)^{n}}\right)
\end{aligned}
$$

for all $x_{1}, x_{2}, \cdots, x_{n} \in \mathcal{X}$ and all nonnegative integers $n$. Since (2.7) gives that

$$
\lim _{n \rightarrow \infty} 2^{n} \varphi\left(\frac{x_{1}}{(-2)^{n}}, \frac{x_{2}}{(-2)^{n}}, \cdots, \frac{x_{n}}{(-2)^{n}}\right)=0
$$

for all $x_{1}, x_{2}, \cdots, x_{n} \in \mathcal{X}$, letting $n$ tend to $\infty$ in the above inequality, we have

$$
\left\|A\left(2 x_{1}\right)+A\left(2 x_{2}\right)+\cdots+A\left(2 x_{n}\right)\right\| \leq\left\|t A\left(x_{1}+x_{2}+\cdots+x_{n}\right)\right\|
$$

so $A$ is additive by Lemma 2.1 .

Let $A^{\prime}: \mathcal{X} \rightarrow \mathcal{Y}$ be another additive mapping satisfying (2.8). Since both $A$ and $A^{\prime}$ are additive, we have, for all positive integer $n$

$$
\begin{aligned}
& \left\|A(x)-A^{\prime}(x)\right\| \\
& =2^{n}\left\|A\left(\frac{x}{(-2)^{n}}\right)-A^{\prime}\left(\frac{x}{(-2)^{n}}\right)\right\| \\
& \leq 2^{n}\left(\left\|A\left(\frac{x}{(-2)^{n}}\right)-f\left(\frac{x}{(-2)^{n}}\right)\right\|+\left\|f\left(\frac{x}{(-2)^{n}}\right)-A^{\prime}\left(\frac{x}{(-2)^{n}}\right)\right\|\right) \\
& \leq 2^{n} \widetilde{\varphi}\left(\frac{x_{1}}{(-2)^{n}}, \frac{-x_{2}}{(-2)^{n+1}}, \frac{-x_{3}}{(-2)^{n+1}}, 0, \cdots, 0\right)
\end{aligned}
$$




$$
=\sum_{j=n+1}^{\infty} 2^{j} \varphi\left(\frac{x_{1}}{(-2)^{j-n}}, \frac{-x_{2}}{(-2)^{j+1-n}}, \frac{-x_{3}}{(-2)^{j+1-n}}, 0, \cdots, 0\right)
$$

which goes to zero as $n \rightarrow \infty$ for all $x \in \mathcal{X}$ by (2.7). Therefore, $A$ is a unique additive mapping satisfying (2.8), as desired.

\section{References}

[1] D. H. Hyers, On the stability of the linear functional equation, Proc. Nat. Acad. Sci. 27 (1941), 222-224.

[2] J. R. Lee, C. Park, and D. Y. Shin, Stability of an additive functional inequality in proper $C Q^{*}$-algebras, Bull. Korean Math. Soc. 48 (2011), 853-871.

[3] C. Park, Y. S. Cho, and M. H. Han, Functional inequalities associated with Jordan -von Neumann-type additive functional equations, J. Inequal. Appl. 2007 (2007), Article ID 41820, 13 pages.

[4] S. M. Ulam, A Collection of the Mathematical Problems, Interscience Publ., New York, 1960.

Department of Mathematics Education

Mokwon University

Daejeon 302-729, Republic of Korea

E-mail: math888@naver.com 